\title{
Supersymmetric Runge-Lenz-Pauli vector for Dirac vortex in topological insulators and graphene
}

\author{
Chi-Ken Lu and Igor F. Herbut \\ Department of Physics, Simon Fraser University, Burnaby, British Columbia V5A \\ 1S6, Canada \\ E-mail: chikenl@sfu.ca
}

\begin{abstract}
.
The Dirac mass-vortex at the surface of a topological insulator or in graphene is considered. Within the linear approximation for the vortex amplitude's radial dependence, the spectrum is a series of degenerate bound states, which can be classified by a set of accidental $S U(2)$ and supersymmetry generators (Herbut I F and $\mathrm{Lu}$ C K 2011 Phys. Rev. B 83 125412). Here we discuss further the properties and manifestations of the supersymmetry of the vortex Hamiltonian, and point out some interesting analogies with the Runge-Lenz-Pauli vector in the non-relativistic hydrogen atom. Symmetry breaking effects due to a finite chemical potential, and the Zeeman field are also analyzed. We find that a residual accidental degeneracy remains only in the special case of equal magnitudes of both terms, whereas otherwise it becomes removed entirely.
\end{abstract}

PACS numbers: 03.65.Ge,11.30.Pb,74.45.+c 


\section{Introduction}

Accidental degeneracy has been an interesting issue in mathematical physics, with the classic examples ranging from non-relativistic hydrogen atom to multi-dimensional harmonic oscillator. The fact that states labeled by different quantum numbers share the same energy is explained by identifying hidden constants of motion, unrelated to spatial symmetries that may be present in the problem. In hydrogen atom, for example, these are the three Runge-Lenz-Pauli operators which, together with the usual angular momentum, constitute a closed $s o(3) \times s o(3)$ Lie algebra, and so imply the degeneracy among all the levels of a given principle quantum number. [1, 2] For the hydrogen spectra in the relativistic limit, however, the linear dispersion in the Dirac Hamiltonian together with its matrix structure complicate the degeneracy pattern and hinder the search for the hidden accidental constants of motion. [3, 4]

Recently, the interface between topologically trivial and non-trivial insulator has become a topic of many investigations, because of its hosting of the quasi-relativistic chiral Dirac fermions. [5] Depositing a type-II superconductor onto the surface of a topological insulator, for example, could by the proximity effect induce a mass-term for the Dirac fermions, proportional to the superconducting order parameter. [6] It is well known that, in general, in the vortex configuration of such a mass-term, there exists a special topologically protected state in the Dirac spectrum at zero energy. [7] Many authors have recently elaborated on the properties of such zero-modes in condensed matter systems, and derived several unexpected consequences of their existence. 8, 9, 10, 11, 12, 13, 14, 15, 16,

Obtaining the entire bound spectrum for a general vortex profile $\Delta(r)$, however, is typically not possible analytically, and one has to resort to numerical methods. [17] Nevertheless, the corresponding Dirac Hamiltonian becomes analytically solvable once the amplitude of the vortex is linearized near the origin. [18, 19] In our previous paper, [19] we showed that due to the presence of a closed Clifford algebra associated with Dirac matrices the energy spectrum even in this "harmonic approximation" is non-trivial, and, in particular, exhibits degeneracies due to the hidden and accidental $S U(2)$ and supersymmetries. The eigenvalues are given by $E= \pm \omega \sqrt{N}$, with an energy scale $\omega$, and with integer $N$ corresponding to the total number of fictitious fermions and bosons that can be formally defined in the problem, and then distributed over two (in two dimensions) quantum states in all possible ways. The associated degeneracy then comes out to be $2 N$, and when $N>1$ there are two distinct multiplets of the effective "angular momentum", with $j_{>}=N / 2$ and $j_{<}=N / 2-1$, reminiscent of the nonrelativistic spectrum of the hydrogen atom. [1, 2] Such a bound-state spectrum could be observable by the scanning tunneling microscopy, and would determine the specific heat of the vortex, for example.

In this paper, we investigate further the properties and the algebra of the supersymmetry generators, which are responsible for the degeneracy between different multiplets of the angular momentum. These generators commute with the square of the 
Dirac vortex Hamiltonian, and their emergence in the problem is closely related to the existence of different square roots of the identity matrix. We also establish a partial analogy with the Runge-Lenz-Pauli vector for the text-book hydrogen atom. [1, 2] Besides its relevance to topological insulators, our study is also pertinent to the insulating or superconducting graphene, where the low-energy excitations are also Dirac fermions, and the mass-gap can in principle be generated dynamically, as well as externally . 10, 11, 14]

In addition to the usual symmetry of the Dirac spectrum around zero energy, the chiral symmetry of the Dirac Hamiltonian is also responsible for the appearance of the linear combination of odd and even occupation numbers of the fictitious fermions in the bound-state wave functions. We use this property to investigate the symmetry breaking effects of the chemical potential and the Zeeman magnetic field, which in reality are present both in graphene and in topological insulators. We find that the degeneracy is completely lifted in general, but some residual degeneracy remains in the special case when the two contributions are equal in strength.

The paper has the following organization. In Sec. 2, the supersymmetry generators and their superalgebra are introduced in terms of the associated supercharge. Sec. 3 is devoted to explicitly constructing these symmetry generators. A comparison with the Runge-Lenz-Pauli vector is also provided. The effects of the finite chemical potential and the Zeeman field, and their connection with the chiral operator are investigated in Sec. 4. Finally, the discussion of validity of the linear approximation made for the vortex amplitude, and comments on related problems in the literature are offered in Sec. 5. Some explicit wave functions are exhibited in Sec. 6.

\section{Emergent supersymmetry from $H^{2}$}

We define the vortex configuration

$$
\Delta(\vec{r})=|\Delta(r)|(\cos \theta, \sin \theta)
$$

in the two-component order parameter $\Delta(\vec{r})$, which when uniform provides a mass-gap for the two-dimensional Dirac fermions. Such an order parameter may in principle arise from the proximity-induced superconductivity in both graphene and topological insulator, for example. $(r, \theta)$ are the usual polar coordinates in the plane. The winding number of the vortex is assumed to be unity. The spatial profile of the the order parameter's amplitude will be taken to be qualitatively similar to the simple $|\Delta(r)|=\Delta_{\infty} \tanh (r / \xi)$, which features the generic linear dependence on the radial coordinate near the origin and the saturation to a finite value $\Delta_{\infty}$ at infinity. $\xi$ is the characteristic (coherence) length.

With the above qualifications in mind, the linearized Dirac Hamiltonian with the mass-vortex may be written as

$$
H=c\left(\alpha_{1} p_{1}+\alpha_{2} p_{2}\right)+\frac{\Delta_{\infty}}{\xi}\left(\beta_{1} x_{1}+\beta_{2} x_{2}\right)
$$


where the set of four $4 \times 4$ Hermitian matrix $\left\{\alpha_{i}, \beta_{i}\right\}, i=1,2$, constitutes the 4 dimensional Clifford algebra. The fifth matrix $\Gamma=\alpha_{1} \alpha_{2} \beta_{1} \beta_{2}$ is responsible for the chiral symmetry, since it evidently anticommutes with the Hamiltonian: $\{\Gamma, H\}=0$.

A supersymmetric representation of the Dirac Hamiltonian in Eq. (22) is possible both because of the existence of the standard bosonic representation for the operators of coordinates and momenta,

$$
b_{i}=\sqrt{\frac{\Delta_{\infty}}{2 c \xi}} x_{i}+i \sqrt{\frac{c \xi}{2 \Delta_{\infty}}} p_{i}
$$

and of the fermionic representation for the four Dirac matrices, 19]

$$
a_{i}=\frac{\beta_{i}+i \alpha_{i}}{2} .
$$

Here $\left[b_{i}, b_{j}^{\dagger}\right]=\left\{a_{i}, a_{j}^{\dagger}\right\}=\delta_{i j},\left[b_{i}, b_{j}\right]=\left\{a_{i}, a_{j}\right\}=0$, as usual. The two transformations together yield the following supersymmetric Hamiltonian,

$$
H=\omega\left(a_{1}^{\dagger} b_{1}+a_{2}^{\dagger} b_{2}+a_{1} b_{1}^{\dagger}+a_{2} b_{2}^{\dagger}\right),
$$

where the energy $\omega=\sqrt{2 c \Delta_{\infty} / \xi}$. Hereafter, $\omega$ will be assumed to be unity, and will not be shown explicitly unless needed. The energy eigenvalues in this representation become obvious, because

$$
H^{2}=\sum_{i=1,2}\left(a_{i}^{\dagger} a_{i}+b_{i}^{\dagger} b_{i}\right)
$$

turns out to be a simple sum of fermion and boson particle numbers. Therefore, the energy levels can be labeled by the total particle number $N$, and various ways of distributing these fictitious particles according to their statistics leads to the degeneracy of $2 N$. [19] It is also easy to see that the zero-mode in this representation corresponds to the nondegenerate vacuum $|0\rangle$, which is also an eigenstate of the chiral symmetry operator $\Gamma$ with the eigenvalue of unity.

In order to see how the underlying supersymmetry emerges, we first note the commutators $\left[H^{2}, a_{i}^{\dagger}\right]=a_{i}^{\dagger}$ and $\left[H^{2}, b_{i}^{\dagger}\right]=b_{i}^{\dagger}$ for $i=1,2$. We then search for other operators whose square has the identical commutation relations with the particle operators. With the help of identity $\left[A^{2}, X\right]=\{A,[A, X]\}=[A,\{A, X\}]$ for either $X=b_{i}^{\dagger}$ or $X=a_{i}^{\dagger}$, one readily finds the following three such additional supersymmetry operators:

$$
A_{i}=\sum_{j, k=1,2} \sigma_{i, j k}\left(a_{j}^{\dagger} b_{k}+b_{j}^{\dagger} a_{k}\right),
$$

which satisfy $A_{i}^{2}=H^{2}$ (without the summation convention). In the above, the index $i$ denotes the i-th Pauli matrix, whereas the subscripts $(j, k)$ label the matrix elements. The existence of these operators is linked to the fact that the two-by-two identity matrix $I_{2}$ has four distinct square roots. Together with $H$, the four supersymmetry operators may be decomposed into the supercharges and their Hermitian conjugates, 20] 
$(H, \vec{A})=Q_{\mu}+Q_{\mu}^{\dagger}$. The time-component $\mu=0$ corresponds to $H$ and the rest of components refers to $\vec{A}$. The supercharge is defined as

$$
Q_{\mu}=\sum_{j, k=1,2} b_{j}^{\dagger}\left(\sigma_{\mu}\right)_{j k} a_{k}
$$

We now determine the algebra satisfied by the supersymmetry operators. First, due to the quantum statistics associated with the $a$ 's and $b$ 's, the supercharges are mutually anticommuting:

$$
\left\{Q_{\mu}, Q_{\nu}\right\}=0 \text {. }
$$

Next, it is useful to invoke the anticommutator

$$
\left\{a_{i}^{\dagger} b_{j}, b_{l}^{\dagger} a_{m}\right\}=a_{i}^{\dagger} a_{m} \delta_{j l}+b_{l}^{\dagger} b_{j} \delta_{i m},
$$

between boson-fermion mixed operators to compute the anticommutators between $Q$ 's and $Q^{\dagger}$ 's,

$$
\left\{Q_{\mu}, Q_{\nu}^{\dagger}\right\}=\sum_{j, k=1,2}\left(b_{j}^{\dagger}\left(\sigma_{\mu} \sigma_{\nu}\right)_{j k} b_{k}+a_{j}^{\dagger}\left(\sigma_{\nu} \sigma_{\mu}\right)_{j k} a_{k}\right)
$$

which will be crucial in the subsequent generation of the constants of motion. Eq. (11) leads to the following simple superalgebra for the components of the operator $\vec{A}$ :

$$
\left\{A_{i}, A_{j}\right\}=2 H^{2} \delta_{i j} .
$$

When the time-component $H$ is involved in Eq. (11), on the other hand, one obtains the generators of the $S U(2)$ symmetry of the Dirac Hamiltonian as

$$
\{H, \vec{A}\}=4 \vec{J},
$$

defined as, 19

$$
J_{i}=\frac{1}{2} \sum_{j, k=1,2} \sigma_{i, j k}\left(a_{j}^{\dagger} a_{k}+b_{j}^{\dagger} b_{k}\right) .
$$

From Eq. (12) and (13), we then see that $\vec{J}$ is a constant of motion, since

$$
\left[J_{i}, H\right]=\frac{1}{4}\left[\left\{H, A_{i}\right\}, H\right]=0 .
$$

Next, the algebra between $J$ 's and $A$ 's may be displayed in terms of commutators, which also make the vector nature of the latter under the $S U(2)$ symmetry manifest. The following identity,

$$
\left[X_{i}^{\dagger} X_{j}, X_{l}^{\dagger} X_{m}\right]=X_{i}^{\dagger} X_{m} \delta_{j l}-X_{l}^{\dagger} X_{j} \delta_{i m},
$$

for both fermion $X=a$ and boson $X=b$ can be used to derive the standard $S U(2)$ commutator algebra between the angular momentum generators,

$$
\left[J_{i}, J_{j}\right]=i \epsilon_{i j k} J_{k} .
$$

From the commutator between distinct species,

$$
\left[X_{i}^{\dagger} Y_{j}, Y_{l}^{\dagger} Y_{m}\right]=X_{i}^{\dagger} Y_{m} \delta_{j l}
$$


for $X=a(b)$ and $Y=b(a)$, on the other hand, one can show that $\vec{A}$ is indeed a vector under the $S U(2)$ algebra, i. e.

$$
\left[A_{i}, J_{j}\right]=i \epsilon_{i j k} A_{k} .
$$

Eqs. (12), (13), (17) and (19) constitute the superalgebra that is behind the degeneracy of the spectrum of $H$. The reader should note that Eq. (13), (17) and (19) are mutually consistent: this can be seen, for example, through replacing with $J_{1}=\left\{H, A_{1}\right\} / 4$ to show that $\left[J_{1}, J_{2}\right]=\left\{\left[A_{1}, J_{2}\right], H\right\} / 4=i J_{3}$. In order to include the chiral symmetry operator $\Gamma$ and complete the algebra, we note that $\left\{\Gamma, a_{i}\right\}=\left[\Gamma, b_{i}\right]=0$, which yields the final relations,

$$
\begin{aligned}
& \{\Gamma, H\}=\{\Gamma, \vec{A}\}=0, \\
& {[\Gamma, \vec{J}]=0}
\end{aligned}
$$

Let us remark in passing that the above derivation can be extended to higher dimensions, to consider, for example, the three-component mass hedgehog in the threedimensional Dirac equation. [16, 19, 21] This case would involve distributing fermions and bosons over three quantum states to obtain the spectral degeneracies. The essential modification would then be the replacement of the matrices $\left\{I_{2}, \vec{\sigma}\right\}$ which appear in Eq. (7) and (14) with another set, consisting of an identity matrix and the eight GellMann matrices. 222 Again, the equalities Eq. (10), (16), and (18) are still valid between three boson and three fermion operators. Therefore, the commutation relations in Eq. (17) and (19) still hold, except that the structure factor associated with $S U(3)$ algebra should be involved. Although $\left[A_{i}^{2}, H^{2}\right]=0$ also holds here, $A_{i}^{2}$ does not coincide with $H^{2}$ as in $S U(2)$ case. Nevertheless, the square of Hamiltonian still can be expressed as the sum $H^{2}=(3 / 32) \sum_{i=1}^{8} A_{i}^{2}$.

\section{Representation of supersymmetry operators and connection with Runge-Lenz-Pauli vector}

The degeneracy pattern of the spectrum of the Hamiltonian in Eq. (2) (Fig.1 in Ref.[19]) is similar to that of the bound spectrum in hydrogen. It is therefore of interest to investigate how the states within each eigenspace with $E=\sqrt{N}$ are connected to each other via the application of the operators $\vec{J}$ and $\vec{A}$. In addition, since the subspace of the second excited level with $N=2$ has a one-to-one correspondence with that of the first excited level with $n=2$ in the hydrogen atom, we should be able to see the exact correspondence in algebraic relations between the two cases.

First, a matrix representation for vector operator $\vec{A}$ of Eq. (7) will be constructed. We work in the basis of eigenstates $|E, j, m\rangle$ labelled by the respective eigenvalues of $H, J^{2}$ and $J_{3}$. We recall that for $N>1$ there are two allowed magnitudes of angular

momentum $j_{>}=N / 2$ and $j_{<}=j_{>}-1$ within the degenerate subspace with $E=\sqrt{N}$. [19] The resultant degeneracy is then $2 N$. The chiral operator $\Gamma$ also connects the states with opposite signs of the energy, as usual. 
According to Eq. (19), $A_{3}$ commutes with $J_{3}$ and $H^{2}$. When $N>1$, it suffices then to consider the subset consisting of the four states $\left\{\left| \pm E, j_{>}, m\right\rangle,\left| \pm E, j_{<}, m\right\rangle\right\}$. We now show that:

$$
A_{3}\left|E, j_{>}, m\right\rangle=\frac{2 m}{E}\left|E, j_{>}, m\right\rangle+\sqrt{E^{2}-\frac{4 m^{2}}{E^{2}}}\left|-E, j_{<}, m\right\rangle .
$$

First, bracketing $\left\{H, A_{3}\right\}=4 J_{3}$ between a pair of states leads to

$$
\left\langle E, j_{>}, m\left|A_{3}\right| E, j_{>}, m\right\rangle=\frac{2 m}{E},
$$

and to the conclusion that $A_{3}\left|E, j_{>}, m\right\rangle$ is orthogonal to $\left|E, j_{<}, m\right\rangle$. Next, due to Eq. (20), the matrix element

$$
\left\langle-E, j_{>}, m\left|A_{3}\right| E, j_{>}, m\right\rangle=-\left\langle-E, j_{>}, m\left|A_{3}\right| E, j_{>}, m\right\rangle^{*}
$$

must be purely imaginary, or zero. Since $A_{3}$ in Eq. (77) has only real coefficients, one concludes that $A_{3}\left|E, j_{>}, m\right\rangle$ is orthogonal to $\left|-E, j_{>}, m\right\rangle$ as well. Consequently,

$$
A_{3}\left|E, j_{>}, m\right\rangle=\frac{2 m}{E}\left|E, j_{>}, m\right\rangle+d\left|-E, j_{<}, m\right\rangle
$$

with a yet undetermined coefficient $d$. Similarly,

$$
A_{3}\left|-E, j_{<}, m\right\rangle=-\frac{2 m}{E}\left|-E, j_{<}, m\right\rangle+d\left|E, j_{>}, m\right\rangle .
$$

Using then $A_{3}^{2}=H^{2}$ fixes the value of the coefficient $d$ to $d^{2}=E^{2}-4 m^{2} / E^{2}$, as in Eq. (22). Utilizing the commutators $\left[A_{3}, J_{ \pm}\right]= \pm A_{ \pm}$would then further provide the matrix forms of $A_{1}$ and $A_{2}$, for example.

A compact representation for the operators $\vec{A}$ is available within the degenerate subspace of dimension $4 N$ and corresponding to $E^{2}=N$, which is the orthogonal sum of the two energy eigenspaces with the positive and negative energies of equal magnitude. It is straightforward to write down the operators $H$ and $\vec{J}$ as block-diagonal matrices,

$$
\begin{aligned}
& \frac{H}{\sqrt{N}}=\sigma_{3} \otimes I_{2 N}, \\
& J_{i}=I_{2} \otimes L_{i} .
\end{aligned}
$$

The identity matrix $I_{2 N}$ and the projected (reducible) angular momentum matrices $\vec{L}$ act within the degenerate subspace of $E=\sqrt{N}$, and of $E=-\sqrt{N}$. The chiral symmetry operator $\Gamma$ that has the effect of reversing the sign of energy can be similarly expressed as

$$
\Gamma=\sigma_{1} \otimes I_{2 N}
$$

The vector operator $\vec{A}$ projected onto the $E^{2}=N$ subspace may be seen to possess the form,

$$
A_{i}=\frac{2}{\sqrt{N}} \sigma_{3} \otimes L_{i}+2 \sqrt{N} \sigma_{2} \otimes K_{i}
$$

in which through Eq. (13) the first term generates the above $\vec{J}$. The second term contains yet another vector $\vec{K}$, which due to the multiplication by $\sigma_{2}$ cancels out in 
Eq. (13), but will be important for the analogy with the Runge-Lenz-Pauli vector to be drawn shortly. Via Eq. (19) we then find

$$
\left[K_{i}, L_{j}\right]=i \epsilon_{i j k} K_{k}
$$

which resembles the commutation relation between the components of the Runge-LenzPauli vector and the orbital angular momentum in the hydrogen atom. [2] Moreover, the coincidence between $H^{2}$ and $A_{i}^{2}$ implies the following identity,

$$
\frac{1}{N^{2}} L_{i}^{2}+K_{i}^{2}=\frac{1}{4} I_{2 N}
$$

to be true for all $i=1,2,3$. Consequently, the sum of squares for both vectors turns into $L^{2} / N^{2}+K^{2}=3 / 4$. In the hydrogen's bound spectrum, on the other hand, the corresponding identity reads $K^{2}+L^{2} / n^{2}=1-1 / n^{2},[2]$ where the principle quantum number is denoted by $n$. The two identities indeed match, but only when the respective degenerate subspaces contain two angular momentum multiplets. In hydrogen this is only true for the energy eigenspace with $n=2$, which is $2 s \oplus 2 p$. In addition, one can also rewrite the anticommutators in Eq. (12) in terms of the vectors $\vec{L}$ and $\vec{K}$ as,

$$
\left\{A_{i}, A_{j}\right\}=I_{2} \otimes\left(\frac{4}{N}\left\{L_{i}, L_{j}\right\}+4 N\left\{K_{i}, K_{j}\right\}\right),
$$

from which the pair of anticommutators adds up to zero for different indices. For example, $\left\{K_{1}, K_{2}\right\}=(-i)\left\{\left[K_{2}, L_{3}\right], K_{2}\right\}=\left(i / N^{2}\right)\left\{\left[L_{2}, L_{3}\right], L_{2}\right\}$ is obtained through utilizing Eq. (31) and (32), and therefore the above sum of anticommutators vanishes.

\section{Effects of chemical potential and Zeeman field}

In this section we study the effects of chemical potential and of the Zeeman coupling to the external magnetic field perpendicular to the surface of the topological insulator. At the surface of topological insulator, the spin-rotational invariance is broken by the kinetic energy term in Eq. (2), which suggests that the Hermitian matrix $i \alpha_{1} \alpha_{2}$ should correspond to the out-of-plane component of the spin. Since the two mass-terms are rotated into each other by the matrix $i \beta_{1} \beta_{2}$, we recognize this operator as representing the particle number, i. e. the generator of the $U(1)$ symmetry associated with the superconducting phase. Therefore, the out-of-plane Zeeman field $h$ and chemical potential $\mu$ shall enter the Hamiltonian through the couplings to $i \alpha_{1} \alpha_{2}$ and $i \beta_{1} \beta_{2}$, respectively. It will be useful, however, to represent these two terms by their sum $\Psi$ and their difference $\Pi$ instead:

$$
\Psi \equiv \frac{i}{2}\left(\beta_{1} \beta_{2}+\alpha_{1} \alpha_{2}\right)=i\left(a_{1}^{\dagger} a_{2}-a_{2}^{\dagger} a_{1}\right)
$$

and

$$
\Pi \equiv \frac{i}{2}\left(\beta_{1} \beta_{2}-\alpha_{1} \alpha_{2}\right)=i\left(a_{1}^{\dagger} a_{2}^{\dagger}+a_{1} a_{2}\right)
$$


We notice that the operator $\Psi$ coincides with the fermion part of angular momentum operator $J_{2}$ in Eq. (14), so $\left[\Psi, J_{2}\right]=\left[\Psi, H^{2}\right]=0$. The operator $\Pi$, on the other hand, is a fermion "pairing" term, which is also $S U(2)$ invariant:

$$
[\Pi, \vec{J}]=0 \text {. }
$$

The chemical potential and the Zeeman coupling then can be written together as $\varepsilon \Pi+\delta \Psi$ with $\varepsilon=\mu-h$ and $\delta=\mu+h$. Before proceeding further, we exploit the $S U(2)$ invariance of the Hamiltonian in Eq. (2) and rotate for convenience the operator $\Psi$ into

$$
\Psi=a_{1}^{\dagger} a_{1}-a_{2}^{\dagger} a_{2}
$$

which is the fermion piece of $J_{3}$.

Since $\Psi$ and $\Pi$ act on the states by changing only the fermion part of wave functions, they can be viewed as two linear and orthogonal operators within the fermion subspace $\left\{|0\rangle, a_{1}^{\dagger}|0\rangle, a_{2}^{\dagger}|0\rangle, a_{1}^{\dagger} a_{2}^{\dagger}|0\rangle\right\}$, in which the common, but for the present purposes irrelevant boson part is not shown explicitly. $\Pi$ projects onto the subspace $\left\{|0\rangle, a_{1}^{\dagger} a_{2}^{\dagger}|0\rangle\right\}$ consisting of even number of fermions, since obviously $\Pi a_{1(2)}^{\dagger}|0\rangle=0$. Moreover,

$$
\begin{aligned}
& \Pi|0\rangle=i a_{1}^{\dagger} a_{2}^{\dagger}|0\rangle, \\
& \prod a_{1}^{\dagger} a_{2}^{\dagger}|0\rangle=-i|0\rangle,
\end{aligned}
$$

so $\Pi$ acts like the $\sigma_{2}$ within this subspace. $\Psi$, in contrast, is a projection operator onto the subspace $\left\{a_{1}^{\dagger}|0\rangle, a_{2}^{\dagger}|0\rangle\right\}$ consisting of only one fermion:

$$
\begin{aligned}
& \Psi a_{1}^{\dagger}|0\rangle=a_{1}^{\dagger}|0\rangle, \\
& \Psi a_{2}^{\dagger}|0\rangle=-a_{2}^{\dagger}|0\rangle,
\end{aligned}
$$

$\Psi$ therefore acts analogously to $\sigma_{3}$ in this subspace.

We may also introduce the projection operators from the chiral symmetry operator $\Gamma$, namely,

$$
\mathcal{P}_{ \pm}=(1 \pm \Gamma) / 2
$$

because $\Gamma$ anticommutes with both fermion operators $a_{1,2}$. This can be seen, for example, from the fact that $\mathcal{P}_{+} a_{1}^{\dagger}|0\rangle=0$ and $\mathcal{P}_{-} a_{1}^{\dagger}|0\rangle=a_{1}^{\dagger}|0\rangle$ since the zero-mode has $\Gamma|0\rangle=|0\rangle$. It follows that $\Psi \mathcal{P}_{+}=\Pi \mathcal{P}_{-}=0$, which can also be seen from the direct computation that yields

$$
\Pi^{2}=\mathcal{P}_{+}, \Psi^{2}=\mathcal{P}_{-} .
$$

The full Hamiltonian including the perturbations can therefore be written as,

$$
H^{\prime}=H+\mathcal{P}_{+}(\varepsilon \Pi) \mathcal{P}_{+}+\mathcal{P}_{-}(\delta \Psi) \mathcal{P}_{-} .
$$

It is easier to consider the effects of $\Pi$ and $\Psi$ separately. Since it commutes with $J_{3}$ and $H^{2}$, the state $|E, j, m\rangle$ is by $\Psi$ coupled only to the state with the same $m$ and the same $E^{2}$. As far as the coupling matrix for $\Psi$ in Eq. (37) is concerned, the only relevant 

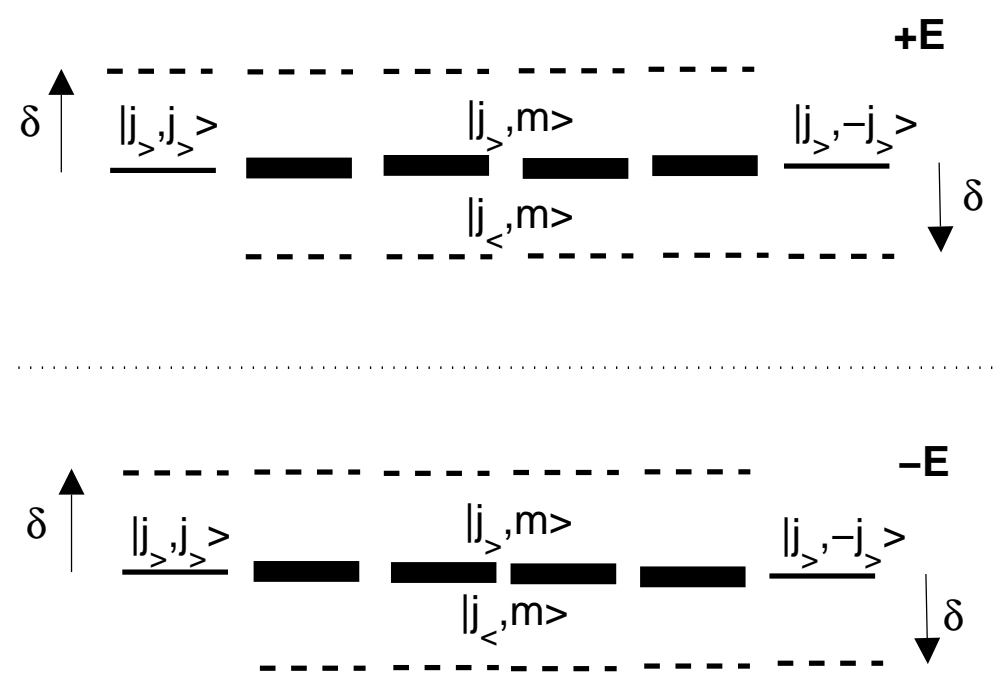

Figure 1. The splitting of the degenerate multiplets $\left|E, j_{>}, m\right\rangle$ and $\left|E, j_{<}, m\right\rangle$ due to the effects of $\Psi$ in Eq. (43), for $N>1$ and $\mu=h=\delta / 2$. The thick solid lines stand for the two states with the same quantum number $m$ but distinct $j$, which are coincident in energy for $\delta=0$. The thin solid lines denote the unique states of maximal value of $|m|$ within this degenerate subspace. Dashed line are the shifted energies when $\delta \neq 0$. The middle dotted line stands for the unperturbed zero energy state.

states are $|1(3)\rangle \equiv\left| \pm E, j_{>}, m\right\rangle$ and $|2(4)\rangle \equiv\left| \pm E, j_{<}, m\right\rangle$ when $|m|<j_{>}$. On the other hand, in the case of largest $|m|=j_{>}$, the relevant states only include $|1\rangle$ and $|3\rangle$. It will be shown shortly that $H+\delta \Psi$ is in this basis reduced to the universal matrix,

$$
E \sigma_{3} \otimes I_{2}+\delta\left(I_{2}-\sigma_{1}\right) \otimes \sigma_{3}
$$

when $|m|<j_{>}$. The two-dimensional matrix,

$$
E \sigma_{3} \pm \delta\left(I_{2}-\sigma_{1}\right)
$$

on the other hand, is responsible for the cases of $m= \pm j_{>}$. In either case, the universal matrix is independent of $m$, and the eigenvalues are $\pm \sqrt{E^{2}+\delta^{2}} \pm \delta$. To the first order of $\delta$, the splitting of the spectrum's degeneracy is shown in Fig, 1 ,

Let us explain the appearance of the above universal matrix in more detail. First consider $|m|<j_{>}$. Recall that $\Psi$ acts only on the one-fermion part of wave functions. Hence, only the projected wave functions,

$$
\mathcal{P}_{-}| \pm E, j, m\rangle= \pm\left[c_{1(2)} f_{m}\left(b_{1}^{\dagger}, b_{2}^{\dagger}\right) a_{1}^{\dagger} \pm c_{2(1)} g_{m}\left(b_{1}^{\dagger}, b_{2}^{\dagger}\right) a_{2}^{\dagger}\right]|0\rangle
$$

are needed when considering the effective couplings. The above $f_{m}$ and $g_{m}$ are some general products of boson operators $b^{\dagger}$ 's that will not affect the following analysis. Within the bracket, the order of coefficients $c_{1}$ and $c_{2}$ as well as \pm sign is to denote the two orthogonal states corresponding to $j=j_{>}$and $j=j_{<}$, respectively, whereas 
the \pm sign preceding the bracket is to denote the sign of $E$. Now it is straightforward to show that $\Psi$ is given by,

$$
\delta \Psi=\delta\left(I_{2}-\sigma_{1}\right) \otimes S
$$

in which the minus sign preceding $\sigma_{1}$ appears because opposite signs are associated with opposite-energy states in Eq. (46). The 2-by-2 matrix $S$ is associated with coupling between $|1\rangle$ and $|2\rangle$. Explicitly,

$$
S=\left(c_{1}^{2}-c_{2}^{2}\right) \sigma_{3}+2 c_{1} c_{2} \sigma_{1}
$$

which yields a pair of eigenvalues $\pm\left(c_{1}^{2}+c_{2}^{2}\right)$. We note that $c_{1}^{2}+c_{2}^{2}=1$ is dictated by the orthogonality between wave functions in Eq. (46). Moreover, we can rotate the space spanned by $|1\rangle$ and $|2\rangle$ so that $S$ turns into a diagonal $\sigma_{3}$, which finally leads to the matrix form in Eq. (44). Similar method can be applied to $|m|=j_{>}$to obtain Eq. (45).

Next we consider the coupling matrix for $\Pi$ in Eq. (35) and the ensuing splitting of the spectrum. Since $[\Pi, \vec{J}]=0$ and $[\Pi, H] \neq 0$, it suffices to consider another subspace consisting of $|1(2)\rangle \equiv\left| \pm E_{1}, j, m\right\rangle$ and $|3(4)\rangle \equiv\left| \pm E_{2}, j, m\right\rangle$ for a given $j$ with the constraint $E_{1}^{2}=E_{2}^{2}+2$. Note that $\Pi$ will only act on the even-fermion part of wave functions $\mathcal{P}_{+}\left|E_{1(2)}, j_{<(>)}, m\right\rangle$. Explicitly, the one including both fermions $a_{1}^{\dagger} a_{2}^{\dagger} u_{m}\left(b_{1}^{\dagger}, b_{2}^{\dagger}\right)|0\rangle$ corresponds to $(E, j)=\left(E_{1}, j_{<}\right)$, while the one without any fermion $u_{m}\left(b_{1}^{\dagger}, b_{2}^{\dagger}\right)|0\rangle$ is responsible when $(E, j)=\left(E_{2}, j_{>}\right)$. As can be seen in the Appendix, $u_{m}$ represents a general product of boson operators $b^{\dagger}$ 's, which will neither affect the coupling matrix of $\Pi$. Then, the four-dimensional matrix representing $H+\varepsilon \Pi$ assumes the following universal form,

$$
E_{s} I_{2} \otimes \sigma_{3}+E_{d} \sigma_{3} \otimes \sigma_{3}+\varepsilon \sigma_{2} \otimes\left(I_{2}+\sigma_{1}\right)
$$

where the energy is defined through $E_{s(d)}=\left(E_{1} \pm E_{2}\right) / 2$. This matrix then yield two new pairs of eigenvalues, $\left|E_{1}^{\prime}\right|=\left|E_{1}\right| \sqrt{1+|\varepsilon|^{2}}$ and $\left|E_{2}^{\prime}\right|=\left|E_{2}\right| \sqrt{1-|\varepsilon|^{2}}$, respectively. As shown in Fig. 2, the degeneracy between a pair of distinct $j$ is removed, while the accidental $S U(2)$ degeneracy remains unresolved.

The effects of the perturbations under consideration on the zero-mode can also be simply analyzed by using the projection operators. Obviously, due to the projection into odd-fermion subspace, the term proportional to $\delta \Psi$ in Eq. (43) by itself has no effect on the zero-mode. The $\Pi$ term in Eq. (43) can only couple the zero-mode to another pair of rotationally invariant states, i.e. the pair of opposite-energy states of $N=2$ and $j=0$. When the $\Pi$-term alone is present, the corresponding three-dimensional matrix that connects the $j=0$ states is then found to always have a zero eigenvalue. Within the linear approximation, we therefore conclude, the zero-mode is still present in the spectrum in the presence of the chemical potential and the Zeeman field, at the special point $|\mu|=|h|$. This agrees with the more general analysis, [23] which shows that in the presence of chiral-symmetry breaking terms such as $h$ and $\mu$, the zero-mode is implied under very broad conditions by the existence of an antilinear operator that anticommutes with the Dirac vortex Hamiltonian. 


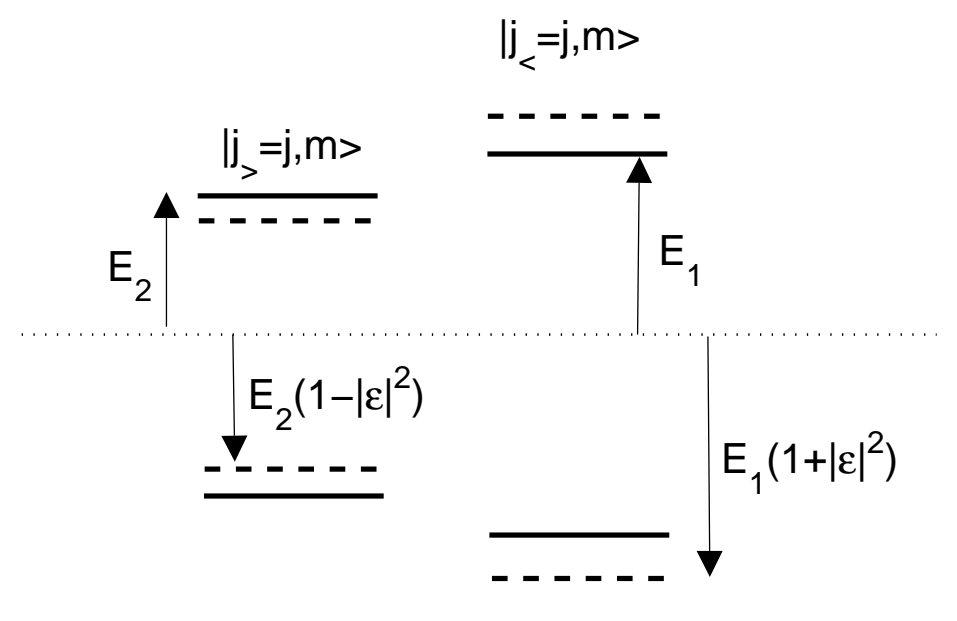

Figure 2. The splitting between the states with the same quantum namber $j$ but with different $E$, due to the $\Pi$-term in Eq. (43). This corresponds to $\mu=-h=\varepsilon / 2$. The solid and dashed lines represent the multiplets before and after, respectively, the extra term $\varepsilon \neq 0$ is turned on. The dotted line in the middle stands for the unperturbed zero-energy state. The degeneracy due to the $S U(2)$ rotational symmetry remains intact in this case.

In presence of both terms $\Psi$ and $\Pi$, the calculation becomes rather complicated, and we will not pursue it further here. As long as the perturbations have weak spatial variation, however, our treatment in terms of fermion operators only is still applicable. Presence of both perturbation in Eq. (43) introduces couplings which are not closed within any finite subset of bound states. For each value of the quantum number $m$, there will be a corresponding matrix of infinite dimension, representing the coupling among all the states with the same $m$. In this case no degeneracy is expected to survive in the spectrum.

\section{Discussions and Conclusions}

The accidental symmetry and the emergent supersymmetry due to $\vec{J}$ and $\vec{A}$ both depend crucially on the assumed linearity as well as on isotropy of the vortex amplitude. Including the higher order terms in $|\Delta(r)|$ would introduce combinations of higher powers of boson operators, which would be expected to remove the accidental degeneracy. The anisotropy in the linear terms, on the other hand, would result in an asymmetry to the square of Hamiltonian $H^{2}=\sum_{i=1,2} m_{i}\left(a_{i}^{\dagger} a_{i}+b_{i}^{\dagger} b_{i}\right)$, [19] and $m_{1} \neq m_{2}$. Following the arguments in Sec. 2, it is evident that finding out the square roots of $H^{2}$ other than $H$ itself is then impossible. Basically, it is due to the fact that the matrix $\left(m_{1}+m_{2}\right) I_{2} / 2+\left(m_{1}-m_{2}\right) \sigma_{3} / 2$ has only one independent square root when the $m_{1} \neq m_{2}$. 
Terms in the Dirac Hamiltonian with the linear dependence on the coordinates may also occur in graphene, for example, in presence of the magnetic field perpendicular to the plane. In this case a rather different supersymmetry was found.[24] Yet another similar but maybe a better known case is the Dirac oscillator 25] with the Hamiltonian

$$
H_{o s}=\alpha_{i}\left(p_{i}-i \Gamma x_{i}\right) .
$$

In this case the crucial difference from our problem lies in that the four Dirac matrices $\left\{\alpha_{1}, \alpha_{2}, i \alpha_{1} \Gamma, i \alpha_{2} \Gamma\right\}$ that appear in $H_{o s}$ do not close a Clifford algebra, i.e. not all the pairs of matrices anticommute. The square of the Hamiltonian, $H_{o s}^{2}$, contains a spinorbit coupling $\left(1-L_{3} S_{3}\right) \Gamma$ in addition to the common part of oscillator Hamiltonian as in our $H^{2}$. Here, the angular momentum $L_{3}=x_{1} p_{2}-x_{2} p_{1}$ and spin $S_{3}=i \alpha_{1} \alpha_{2}$ are defined. Compared to the present problem, the extra term in $H_{o s}^{2}$ can be any integer and half-integer, whereas the extra term $\sum_{i=1,2} a_{i}^{\dagger} a_{i}$ in our problem can have the values $\{0,1,2\}$ only. These subtle differences ultimately lead to a rather different underlying (super)symmetry and the ensuing degeneracies of the spectrum.

By solving the differential equation for the zero-mode's wave-function of our linearized Hamiltonian one finds its radial dependence to be $\exp \left(-\Delta_{\infty} r^{2} / 2 c \xi\right)$. Thus, the linearization of the vortex profile is a good approximation when the coherence length is long, $c \ll \Delta_{\infty} \xi$. Also, in the case of strictly linear vortex amplitude, strong external Zeeman field is not able to remove the zero-mode from the spectrum. This stands in contrast to the previously studied case of an order parameter which saturates to a finite value far from the vortex. 23, 26] The spinor part of zero-mode can be deduced from the matrix equation $a_{1}|0\rangle=a_{2}|0\rangle=0$. Using the previous notations in Ref.[26], the fermion operators have the explicit matrix form,

$$
a_{1}=\left(\begin{array}{cc}
i \sigma_{2} & I_{2} \\
I_{2} & -i \sigma_{2}
\end{array}\right), a_{2}=\left(\begin{array}{cc}
-i \sigma_{1} & i I_{2} \\
-i I_{2} & i \sigma_{1}
\end{array}\right),
$$

which yields the column vector $|0\rangle \propto(1,0,0,1)^{T}$ for the zero-mode.

Lastly, the treatment of chemical potential and Zeeman field with two projections $\Psi$ and $\Pi$ is also relevant to a recent study on two-velocity Weyl fermions realized in two-dimensional optical lattice. [27] The effective low-energy Hamiltonian for the spinless fermions can be written as

$$
H_{W}=k_{x} \alpha_{1}+k_{y} \alpha_{2}+\eta\left(i k_{x} \Gamma \beta_{1}+i k_{y} \Gamma \beta_{2}\right),
$$

which yields the eigenvalues $\pm(1 \pm \eta) k$, and $\eta$ is a number indicating the difference in "speed of light". Actually the above Weyl Hamiltonian can be expressed as a more compact form as one notices that the sets of matrices $U=\left\{\alpha_{1}, \alpha_{2}, i \alpha_{1} \alpha_{2}\right\}$ and $V=\left\{i \Gamma \beta_{1}, i \Gamma \beta_{2}, i \beta_{1} \beta_{2}\right\}$ form two commutating su(2) Lie algebras. Thus a generic Hamiltonian of

$$
H_{g}=\vec{u} \cdot \vec{U}+\vec{v} \cdot \vec{V},
$$

with components $\vec{u}=\left(k_{x}, k_{y}, 0\right)$ and $\vec{v}=\eta\left(k_{x}, k_{y}, 0\right)$, can be co-rotated into $|\vec{u}| U_{3}+|\vec{v}| V_{3}$. Then it is easy to see that $H_{W}=(1+\eta) k \Psi+(1-\eta) k \Pi$, which explains that 
the faster/slower Weyl fermions in fact correspond to the odd-fermion/even-fermion subspaces in our definition.

In conclusion, for the two-dimensional linearized vortex on the superconducting surface of topological insulator, we defined the corresponding supersymmetric RungeLenz-Pauli operator, and discussed the connection to its counterpart in the hydrogen atom. We also studied the effects of the physical perturbations such as finite chemical potential and Zeeman coupling of the spin to the out-of plane magnetic field on the spectrum, and showed how these can be economically accounted for in terms of the even/odd-fermion projection operators which we introduced. In the most general case, the accidental degeneracy of the spectrum is removed by these perturbations, but the energy of the zero-mode is unaltered. Unexpectedly, however, we found that some of the accidental degeneracy remains present when the chemical potential and the Zeeman field have identical magnitudes.

\section{Acknowledgment}

This work has been supported by the NSC Taiwan (C.K.L.) and the NSERC of Canada (I.F.H.).

\section{Appendix}

Within the subspace of states of $E^{2}=N$, the wave functions with the maximum eigenvalue of $J_{3}$ belong to the $j_{>}$-multiplet. Explicitly, the pair of opposite-energy states can be written as,

$$
\left|E= \pm \sqrt{N}, j_{>}, m=\frac{N}{2}\right\rangle=\frac{1}{\sqrt{2}}\left(\frac{\left(b_{1}^{\dagger}\right)^{N}}{\sqrt{N !}} \pm \frac{a_{1}^{\dagger}\left(b_{1}^{\dagger}\right)^{N-1}}{\sqrt{(N-1) !}}\right)|0\rangle .
$$

Within the same multiplet, the states with one lower in $m$ is obtained by applying $J_{-}$ to the above states. The even-fermion part of $\left|E= \pm \sqrt{N}, j_{>}, m=j_{>}-1\right\rangle$ is,

$$
\frac{1}{\sqrt{2}} \frac{b_{2}^{\dagger}\left(b_{1}^{\dagger}\right)^{N-1}}{\sqrt{(N-1) !}}|0\rangle
$$

while the associated odd-fermion part is,

$$
\pm \frac{1}{\sqrt{2}}\left[\sqrt{\frac{N-1}{N}} \frac{a_{1}^{\dagger} b_{2}^{\dagger}\left(b_{1}^{\dagger}\right)^{N-2}}{\sqrt{(N-2) !}}+\frac{1}{\sqrt{N}} \frac{a_{2}^{\dagger}\left(b_{1}^{\dagger}\right)^{N-1}}{\sqrt{(N-1) !}}\right]|0\rangle,
$$

where the above \pm denotes the corresponding sign of energy. In the other multiplet of $j_{<}$, the state $\left|E= \pm \sqrt{N}, j_{<}, m=j_{>}-1\right\rangle$ has the same $m$ as the above one, and they are orthogonal to each other. Therefore, its even-fermion part is given by,

$$
\frac{a_{1}^{\dagger} a_{2}^{\dagger}\left(b_{1}^{\dagger}\right)^{N-2}}{\sqrt{(N-2) !}}|0\rangle,
$$


while the associated odd-fermion part is,

$$
\mp\left[\frac{1}{\sqrt{N}} \frac{a_{1}^{\dagger} b_{2}^{\dagger}\left(b_{1}^{\dagger}\right)^{N-2}}{\sqrt{(N-2) !}}-\sqrt{\frac{N-1}{N}} \frac{a_{2}^{\dagger}\left(b_{1}^{\dagger}\right)^{N-1}}{\sqrt{(N-1) !}}\right]|0\rangle,
$$

in which a reverse sign is used to denote the corresponding positive/negative energies in this multiplet. This choice is necessary for being consistent with the choice of sign

for the unknown $d$ in Eq. (22). One can find out the rest of wave functions within this subspace by successively applying the ladder operator $J_{-}$to the above states in both multiplets.

\section{References}

[1] Jauch J M and Hill E L 1940 Phys. Rev. 57641

[2] Greenberg D F 1966 Am. J. Phys. 34, 1101

[3] Gotfried K and Yan T M 2003 Quantum Mechanics: Fundamentals (Springer)

[4] For recent studies on degeneracy in spectra of relativistic hydrogen atom, see, for example, Katsura H and Aoki H 2006 J. Math. Phys. 47, 032301

[5] For reviews, see, e.g., Hasan M Z and Kane C L 2010 Rev. Mod. Phys. 823045

[6] Fu L and Kane C L 2008 Phys. Rev. Lett. 100096407.

[7] Jackiw R and Rebbi C 1976 Phys. Rev. D 133398 Jackiw R and Rossi P 1981 Nucl. Phys. B 190681

[8] Volovik G E 1993 JETP Lett. 57244

[9] Ivanov D A 2001 Phys. Rev. Lett. 86268

[10] Hou C Y et al 2007 Phys. Rev. Lett. 98186809 2010 Phys. Rev. B 81075427

[11] Herbut I F 2007 Phys. Rev. Lett. 99206404 2010a Phys. Rev. Lett. 104, 066404 2010b Phys. Rev. B 81205429

[12] Jackiw R and Pi S Y 2007 Phys. Rev. Lett. 98266402 2008 Phys. Rev. B 78132104

[13] Wilczek F 2009 Nat. Phys. 5614

[14] Ghaemi et al 2010 Phys. Rev. B 81081403

[15] Bergman D L and Le Hur K 2009 Phys. Rev. B 79184520

[16] Teo J C Y and Kane C L 2010 Phys. Rev. Lett. 104046401

[17] Khaymovich I M et al 2009 Phys. Rev. B 79224506

[18] Seradjeh B 2008 Nucl. Phys. B 805182

[19] Herbut I F and Lu C K 2011 Phys. Rev. B 83125412

[20] For examples, see, e.g., Zee A 2003 Quantum Field Theory in a Nutshell (Princeton: Princeton University Press)

[21] Hosur P et al 2010 Phys. Rev. B 81045120

[22] Georgi H 1999 Lie Algebras in Particle Physics (Boulder: Westview Press)

[23] Herbut I F and Lu C K 2010 Phys. Rev. B 82125402

[24] Ezawa M 2008 Phys. Lett. A 372924

[25] Moshinsky M and Szczepaniak A 1989 J. Phys. A: Math. Gen. 22 L817

[26] Lu C K and Herbut I F 2010 Phys. Rev. B 82144505

[27] Kennett M P et al preprint arXiv:1011.1502. 